\title{
Spontaneous dyskinesia in first episode schizophrenia
}

\author{
B K Puri, Thomas R E Barnes, M J Chapman, S B Hutton, Eileen M Joyce
}

\begin{abstract}
In the west London prospective study of first episode schizophrenia, the prevalence and nature of abnormal involuntary movements were examined in 27 patients who had never received antipsychotic drugs and 36 who had been treated with such medication. Motor disturbance was assessed with rating scales designed to cover the full range of spontaneous and drug induced movement disorder. Only one person in the drug naive group showed evidence of parkinsonism, a finding which contrasts with recent reports suggesting that spontaneous extrapyramidal signs may not be uncommon in such patients. However, according to ratings on the modified Rogers scale, $11 \%$ of the drug naïve group exhibited orofacial dyskinesia, $4 \%$ trunk and limb dyskinesia, $7 \%$ postural abnormalities, and $4 \%$ increased muscle tone. The respective figures in the closely matched medicated group were not significantly different except for increased muscle tone, which was significantly more common (25\%). The proportion of drug naive patients fulfilling criteria for tardive dyskinesia on the abnormal involuntary movements scale ranged from $4 \%$ to $11 \%$ depending on the criterion threshold score used. These findings are in accord with the notion that abnormal involuntary movements, particularly orofacial dyskinesia, represent a neuromotor component of schizophrenia.

(F Neurol Neurosurg Psychiatry 1999;66:76-78)
\end{abstract}

Keywords: schizophrenia; first-episode; orofacial movements; spontaneous dyskinesia

One hypothesis regarding the role of antipsychotic drugs in the development of tardive dyskinesia is that they provoke a tendency to abnormal involuntary movements inherent in some forms of schizophrenia. ${ }^{12}$ More specifically, the pathological processes integral to schizophrenia as well as age related cerebral dysfunction may be predisposing factors for the emergence of tardive dyskinesia during chronic antipsychotic administration. ${ }^{3}{ }^{4}$ If this hypothesis were true, one prediction might be that the illness related dysfunction could lead to abnormal involuntary movements resembling tardive dyskinesia even without antipsychotic exposure. Contemporary evidence to support this notion has been provided by the identification of spontaneous dyskinesia in a proportion of drug naïve patients with schizophrenia. ${ }^{5-9}$ The prevalence of dyskinesia reported ranges from $1 \%$, in a first episode study $^{7}$ to over $50 \%$ in a study of inpatients with chronic schizophrenia. ${ }^{5}$ This broad range partly reflects the variation in the mean ages of the samples and the diagnostic criteria used, the second being most commonly a threshold score on the abnormal involuntary movements scale (AIMS). ${ }^{10}$

This study was undertaken in the context of a prospective, neurobiological study of first episode patients with schizophrenia in west London. The aims were to identify the presence and nature of abnormal involuntary movements in antipsychotic naïve patients at the time of their first presentation to psychiatric services, and compare this group with those first episode patients who had already received antipsychotic drugs.

\section{Patients and methods}

The prevalence and nature of abnormal movements were studied in patients presenting to psychiatric services for the first time with a psychotic illness, usually at the point of hospital admission. Eligible patients were aged between 16 and 50 years, with a diagnosis of schizophrenia or schizophreniform disorder according to DSM-IV diagnostic criteria. ${ }^{11}$ The catchment area for the study comprised inner London areas and a more suburban, outer London area. Ethical approval for the study was obtained from the relevant local ethics committees.

All patients were assessed by two of the authors (BKP and MJC) using the following rating instruments: the modified Rogers scale, ${ }^{12} 13$ designed to rate movement disorder in psychiatric patients avoiding assumptions about a psychiatric or neurological aetiology; the AIMS; the extrapyramidal side-effects rating scale (EPSE) ${ }^{14}$ to rate parkinsonian features; and the Barnes akathisia rating scale $(\text { BARS })^{15}$ 
Movement disorder in neuroleptic naïve and neuroleptic treated patients with first episode schizophrenia

\begin{tabular}{|c|c|c|c|}
\hline & $\begin{array}{l}\text { Neuroleptic treated } \\
(n=36) n(\%)\end{array}$ & $\begin{array}{l}\text { Neuroleptic naïve } \\
(n=27) n(\%)\end{array}$ & $p$ Value \\
\hline \multicolumn{4}{|l|}{ Modified Rogers scale: } \\
\hline Posture & $1(3)$ & $2(7)$ & NS 0.39 \\
\hline Tone & $9(25)$ & $1(4)$ & 0.022 \\
\hline \multicolumn{4}{|l|}{ Abnormal movements: } \\
\hline Face and head & $2(6)$ & $3(11)$ & NS 0.36 \\
\hline Trunk and limbs & $1(3)$ & $1(4)$ & NS 0.68 \\
\hline Abnormal ocular movements & $0(0)$ & $2(7)$ & NS 0.18 \\
\hline Purposeful movements & $2(6)$ & $1(4)$ & NS 0.61 \\
\hline Gait & $4(11)$ & $1(4)$ & NS 0.28 \\
\hline Speech & $3(8)$ & $5(18.5)$ & NS 0.21 \\
\hline \multicolumn{4}{|l|}{ Behaviour: } \\
\hline At interview & $5(14)$ & $5(18.5)$ & NS 0.44 \\
\hline Reported & $3(8)$ & $4(15)$ & NS 0.34 \\
\hline \multicolumn{4}{|l|}{$\begin{array}{l}\text { Tardive dyskinesia criteria: } \\
\quad \geqslant 1 \text { on single AIMS item }\end{array}$} \\
\hline Orofacial & $4(11)$ & $3(11)$ & NS 0.66 \\
\hline Trunk and limb & $4(11)$ & $1(4)$ & NS 0.28 \\
\hline Any body site & $5(14)$ & $3(11)$ & NS 0.53 \\
\hline$\geqslant 1$ on two or more AIMS items 6 & $3(8)$ & $1(4)$ & NS 0.42 \\
\hline$\geqslant 2$ on one or more AIMS items ${ }^{5}$ & $3(8)$ & $2(7)$ & NS 0.64 \\
\hline \multicolumn{4}{|l|}{ Parkinsonism: } \\
\hline EPSE: parkinsonism present & $6(17)$ & $1(4)$ & NS 0.11 \\
\hline
\end{tabular}

Results

Sixty three patients were assessed, 27 of whom had never received antipsychotic medication. These 27 patients did not differ significantly from the 36 patients who had received such treatment by the time of assessment in terms of age (range: 16-47 years (neuroleptic naïve) $v$ 17-48 years (medicated); mean (SD): 27.2 (8.4) years $v 26.7(8.1)$ years, $t=0.23, \mathrm{df}=61$, $\mathrm{p}=0.819$ ), sex (male:female: 18:9 v 24:12, $\chi^{2}=0.073$, df $=1, \mathrm{p}=0.787$ ), or ethnicity (White: AfroCaribbean: other: 14:4:9 v 21:8:7, $\left.\chi^{2}=1.733, \mathrm{df}=2, \mathrm{p}=0.42\right)$. For the 36 medicated patients, the mean duration of exposure to antipsychotic drugs at the time of assessment was 22.8 (SD 21.0) days and the mean chlorpromazine equivalent daily dose of antipsychotic medication was $365 \mathrm{mg}$ (range 75-1000 mg). The mean duration of illness with positive psychotic symptoms, to the nearest month, for the unmedicated patients (1 year 10 months, SD 3 years 9 months) was longer than that for the treated patients ( 8 months, SD 1 year 2 months), but the difference was not significant $(t=1.5$, df 29.6, $\mathrm{p}=0.15)$.

Eight patients showed evidence of movement disorder, defined as a rating for the presence of abnormal movements on the Rogers scale and/or a score of 1 or more on an anatomical item of the AIMS. The mean duration of untreated psychosis for these patients (1 year and 8 months, SD 2 years) was not significantly different from the figure for the 55 patients without movement disorder ( 1 year 1 month, SD 2 years 9 months $)(t=0.535, \mathrm{df}=61$, $\mathrm{p}=0.59)$.

The table provides details of the movement disorder in the two patient groups, as measured on the modified Rogers scale, AIMS, and EPSE. The AIMS data are presented in terms of the proportion of patients fulfilling a range of different diagnostic threshold scores that have been used in published studies ${ }^{56}$ to determine the presence of dyskinesia. No patient fulfilled the criterion for the presence of akathisia, which was a score of 2 or more on the global item of the BARS. Only one patient (4\%) in the never treated group exhibited increased muscle tone, as rated by both the modified Rogers scale and EPSE. This was the only unmedicated patient to reach the diagnostic threshold score on the EPSE for the presence of parkinsonism, compared with six (17\%) of the neuroleptic treated patients (table).

In summary, the ratings on the modified Rogers, AIMS, and EPSE rating scales indicated, with a high level of consistency, the presence of abnormal movements in neuroleptic naïve patients. Specifically, according to ratings on the Rogers scale, $11 \%$ exhibited orofacial dyskinesia, $4 \%$ trunk and limb dyskinesia, $7 \%$ postural abnormalities, and $4 \%$ increased muscle tone. Although the respective prevalence figures were higher in the medicated group, only muscle tone $(25 \%)$ was significantly higher in this group, and then only according to ratings on the modified Rogers scale, not the EPSE.

\section{Discussion}

DYSKINESIA

The findings of previous studies of abnormal involuntary movements in drug free patients with schizophrenia have been inconsistent in terms of the presence and severity of dyskinesia. Some studies have found no evidence of either orofacial or trunk and limb dyskinesia. ${ }^{16-18}$ Others have found only a very low prevalence of dyskinesia. For example, Chatterjee et $a l,^{7}$ using the Simpson tardive dyskinesia scale, ${ }^{19}$ reported spontaneous dyskinesia in just one of a sample of 89 neuroleptic naïve, first episode patients. However, higher prevalence figures have been reported in recent studies. ${ }^{8202122}$ McCreadie et al ${ }^{8}$ reported dyskinesia in $38 \%$ of a sample of 21 elderly patients with schizophrenia who had never received medication. Fenn et $a l^{21}$ found that three out of $22(14 \%)$ never medicated patients with schizophrenia presented movements fulfilling research diagnostic criteria for probable tardive dyskinesia, although a further five (23\%) patients exhibited mild dyskinesia. The same team of investigators ${ }^{20}$ also assessed abnormal involuntary movements in 62 neuroleptic naïve patients with schizophrenia, 42 patients who had received antipsychotic medication for at least a year, and 21 control subjects without psychiatric illness. Significantly more of the neuroleptic naïve patients $(26 \%)$ fulfilled criteria for tardive dyskinesia than the drug treated patients $(19 \%)$, whereas none of the healthy controls met the criteria. However, mean total AIMS scores did not differ between the two patient groups. Fenton et al ${ }^{2}$ carried out a retrospective case note study of psychiatric patients who had never received treatment with antipsychotic medication. Dyskinetic movements were more prevalent in the 94 patients with schizophrenia compared with 179 patients with other diagnoses. In $15 \%$ of the first group there was judged to be convincing documented evidence of orofacial dyskinesia.

In the present sample of first episode patients with schizophrenia, the prevalence of a tardive dyskinesia-like syndrome in the neuroleptic naïve patients ranged from $4 \%$ to $11 \%$, 
depending on the stringency of the diagnostic criteria used (table). In the medicated group, receiving relatively moderate doses of antipsychotic drugs, the respective range was $8 \%$ to $11 \%$. The pattern of the AIMS ratings provided some support for the separation of tardive dyskinesia phenomena into orofacial and trunk and limb subsyndromes. ${ }^{23}$

\section{PARKINSONISM}

Assessing parkinsonism in samples of neuroleptic naïve patients, Caligiuri et $a l^{4}$ reported prevalence figures of $21 \%$ for rigidity and $12 \%$ for bradykinesia, whereas Chatterjee et $a l^{7}$ found that spontaneous extrapyramidal signs were present in $17 \%$. The last group of investigators ${ }^{7}$ suggested that parkinsonism may be intrinsic to the pathophysiology of schizophrenia, and that its expression may be augmented by the administration of a neuroleptic drug. The present study provides only equivocal findings on this point, as parkinsonism was only diagnosed in one neuroleptic naïve patient $(4 \%)$, compared with six $(17 \%)$ of the neuroleptic treated patients, although this difference was not significant in this small sample. Chatterjee et al also concluded that muscle rigidity is the most common extrapyramidal sign in neuroleptic naïve patients with schizophrenia. The findings of the current study lend little support to this notion, as significantly more patients in the medicated group $(25 \%)$ exhibited increased muscle tone according to the modified Rogers scale item, suggesting that this was principally a drug induced phenomenon. Nevertheless, before administering antipsychotic medication to a patient for the first time, careful examination for extrapyramidal signs may be advisable to establish a baseline, and to avoid possible misattribution of such problems to subsequent medication. ${ }^{25}$

\section{Conclusion}

The present study is one of the few to have assessed movement disorder comprehensively in patients presenting with their first episode of schizophrenia. The main finding is that a small proportion of neuroleptic naïe, first episode patients exhibit abnormal involuntary movements-namely, orofacial and trunk and limb dyskinesia, increased muscle tone, and postural abnormalities. Increased muscle tone was the only abnormal motor phenomenon found in the neuroleptic naïve schizophrenic patients that was significantly more common in an age and sex matched control group of neuroleptic treated patients. Thus, movement disorder, particularly orofacial dyskinesia, seems to be part of the clinical presentation of schizophrenia, suggesting that it may be intrinsic to the pathophysiology of the condition.
This study was supported by Wellcome Trust project grant 042025 .

1 Barnes TRE, Liddle PF. Tardive dyskinesia: implications for schizophrenia? In: Schiff AA, Roth M, Freeman HL, eds. Schizophrenia: new pharmacological and clinical development. London: Royal Society of Medicine Services, 1985:81-7.

London: Royal Society of Medicine Services, 1985:81-7.
Waddington JL. Tardive dyskinesia in schizophrenia and other disorders: Associations with ageing, cognitive dysfunction and structural brain pathology in relation to neuroleptic exposure. Human Psychopharmacology 1987;2:1122.

3 Liddle PF, Barnes TRE, Speller JC, et al. Negative symptoms as a risk factor for orofacial dyskinesia. $B r \mathcal{F} P s y-$ chiatry 1993;163:776-80.

4 Collinson SL, Pantelis C, Barnes TRE. Abnormal involuntary movements in schizophrenia and their association with cognitive impairment. In: C Pantelis, HE Nelson, TRE Barnes, eds. Schizophrenia: a neuropsychological perspective. Chichester: John Wiley, 1996:237-58.

5 Owens DGC. Involuntary disorders of movement in chronic schizophrenia: the role of the illness and its treatment. In: Casey DE, Chase TN, Christensen AV, et al, eds. Dyskinesia-Research and treatment (Psychopharmacology Supplementum 2). Berlin: Springer-Verlag, 1985:79-87

6 Caligiuri MP, Lohr JB. A disturbance in the control of muscle force in neuroleptic-naïve schizophrenic patients. Biol Psychiatry 1994;35:104-11.

7 Chatterjee A, Chakos M, Koreen A, et al. Prevalence and clinical correlates of extrapyramidal signs and spontaneous dyskinesia in never-medicated schizophrenic patients. Am $\mathcal{f}$ Psychiatry 1995;152:1724-9.

8 McCreadie RG, Thara R, Kamath S, et al. Abnormal movements in never-medicated Indian patients with schizophrenia. Br F Psychiatry 1996;168:221-6.

9 Fenton WS, Wyatt RJ, McGlashan TH. Risk factors for spontaneous dyskinesia in schizophrenia. Arch Gen Psychiatry 1994;51:643-50.

10 Guy W. ECDEU assessment manual for psychopharmacology, revised edition. Washington, DC: US Department of Health, Education and Welfare, 1976:534-7. (Publication 76-338.)

11 American Psychiatric Association. Diagnostic and statistical manual of mental disorders. 4th edition (DSM-IV). Washington, DC: APA, 1994

12 Rogers D, Karki C, Bartlett C, et al. The motor disorders of mental handicap: an overlap with the motor disorders of severe psychiatric illness. Br F Psychiatry 1991;158:97-102.

13 Lund CE, Mortimer AM, Rogers D, et al. Motor, volitional and behavioural disorders in schizophrenia. I: assessment using the modified Rogers scale. Br F Psychiatry 1991;158: 323-7.

14 Simpson GM, Angus JWS. A rating scale for extrapyramidal side-effects. Acta Psychiatrica Scand 1970;212(suppl 44): $11-19$.

15 Barnes TRE. A rating scale for drug-induced akathisia. $\mathrm{Br} F$ Psychiatry 1989;154:672-6.

16 Chorfi M, Moussaoui D. Never treated schizophrenic patients have no abnormal movements such as tardive dyskinesia. Encephale 1985;11:263-5.

17 McCreadie RG, Ohaeri JU. Movement disorder in never and minimally treated Nigerian schizophrenic patients. $\mathrm{Br}$ f Psychiatry 1994;164:184-9.

18 Hernan Silva I, Jerez CS, Ruiz TA, et al. Lack of involuntary abnormal movements in untreated schizophrenic patients. Actas Luso-Espanolas de Neurologia, Psiquiatria y Ciencias Afines 1994;22:200-2.

19 Simpson GM, Lee JH, Zoubok B, et al. A rating scale for tardive dyskinesia. Psychopharmacology 1979;64:171-9.

20 Hoffman W, Kadri N, Fenn D, et al. Choreo-athetoid movements occur spontaneously in never-medicated patients with schizophrenia. European Neuropsychopharmacology 1996;6(suppl 3):223

21 Fenn DS, Moussaoui D, Hoffman WF, et al. Movements in never-medicated schizophrenics: a preliminary study. Psychopharmacology 1996;123:206-10.

22 Fenton WS, Blyler CR, Wyatt RJ, et al. Comparison of the prevalence of spontaneous dyskinesia in schizophrenic and nonschizophrenic psychiatric patients. $\mathrm{Br} \mathcal{F}$ Psychiatry 1997;171:265-8.

23 Barnes TRE. Movement disorder associated with antipsychotic drugs: the tardive syndromes. Int Rev Psychiatry 1990;2:355-66.

24 Caligiuri MP, Lohr JB, Jeste DV. Parkinsonism in neuroleptic-naïve schizophrenic patients. Am f Psychiatry 1993;150:1343-8.

25 Kopala LC. Spontaneous and drug-induced movement disorders in schizophrenia. Acta Psychiatr Scand 1996;94:1217. 